\title{
LOS BENEFICIOS DEL OCIO JUVENIL Y SU CONTRIBUCIÓN AL DESARROLLO HUMANO ${ }^{1}$ THE BENEFITS OF YOUTH LEISURE AND THEIR CONTRIBUTION TO HUMAN DEVELOPMENT
}

\author{
María Jesús Monteagudo \\ Instituto de Estudios de Ocio \\ Facultad de Ciencias Sociales y Humanas \\ Universidad de Deusto, España \\ mjmonte@deusto.es \\ Ruth Ahedo \\ Instituto de Estudios de Ocio \\ Facultad de Ciencias Sociales y Humanas \\ Universidad de Deusto, España \\ ruth.ahedo@deusto.es \\ Ana Ponce de León \\ Grupo AFYDO \\ Facultad de Letras y de la Educación \\ Universidad de la Rioja, España \\ ana.ponce@unirioja.es
}

\begin{abstract}
Cómo citar / Citation
Monteagudo, María Jesús; Ahedo, Ruth y Ponce de León, Ana (2017) "Los beneficios del ocio juvenil y su contribución al desarrollo humano". OBETS. Revista de Ciencias Sociales, 12(Extra 1): pp-pp. 177-202.
\end{abstract}

doi:10.14198/OBETS2017.12.1.17

\footnotetext{
${ }^{1}$ Esta investigación forma parte del Proyecto "De los tiempos educativos a los tiempos sociales: la participación de los jóvenes en la creación y gobernanza de sus espacios de ocio como factor de desarrollo personal" (RESORTES), financiada en la convocatoria del Plan Nacional de I+D+i (2012), por la Secretaría de Estado de Investigación, Desarrollo e Innovación, adscrita al Ministerio de Economía y Competitividad, con referencia EDU201239080-C07-03.
} 


\title{
Resumen
}

Este artículo analiza los beneficios del ocio juvenil y su aportación al desarrollo personal y social con el objetivo de esclarecer si el tipo de actividad de ocio, el género, los espacios y el carácter espontáneo u organizado del ocio inciden en la percepción de beneficios emocionales, cognitivos, sociales, físicos y desarrollo de destrezas y habilidades técnicas. La muestra se compone de 1253 jóvenes españoles con edades entre los 16 y 18 años. Los resultados corroboran el influjo de estas variables tanto en la percepción de beneficios como en la naturaleza de los mismos. El ocio físico-deportivo no es solo la práctica más significativa, sino también la que les proporciona un abanico más amplio de retornos positivos. Los chicos obtienen puntuaciones más altas en todos los tipos de beneficios, excepto en los emocionales. Algunos espacios del ocio resultan más proclives para el logro de ciertos beneficios. Finalmente, el ocio organizado aporta mayores beneficios que el no organizado. La discusión sobre el rol de estas variables es clave para hacer del ocio un factor de desarrollo juvenil.

Palabras clave: Ocio joven, Beneficios, Tipo de Práctica, Género, Espacios, Organización del ocio.

\begin{abstract}
This study aims to explore the benefits of youth leisure in order to clarify whether the type of leisure activity, gender, space and organization of leisure practice affect the perception of different categories of benefits: emotional, cognitive, social physical and the acquisition of abilities and technical skills. The sample consists of 1253 young Spaniards aged between 16 and 18 years. The results confirm the influence of these variables both in the perception of benefits and nature thereof. Physical-sport leisure is not only the most significant practice, but also the one that gives them a wider range of positive returns. The boys score higher in all types of benefits, except for the emotional. Some spaces of leisure are more prone to the achievement of certain benefits. Finally, organized leisure brings greater benefits than non-organized. The discussion on the role of these variables is key to making leisure a factor of youth development.
\end{abstract}

Key words: youth leisure; benefits; type of practice; gender; space; organization of leisure.

\section{Extended abstract}

This work is part of the coordinated project "From educational time to social time: building the human condition of youth in network societies", whose universe is the young Spanish population aged between 16 and 18 years. The study delves into the benefits of youth leisure, focusing on those leisure practices that are identified as particularly significant, that is, those that are especially important for them, and which they do not want to give up. The study is addressed from the approach of young leisure as a valuable experience, which provides a new perspective to look into the benefits of leisure.

Understanding youth leisure in an experiential way implies recognizing that not all experiences of youth leisure contribute identically to personal and 
social development (Cuenca, 2015, Monteagudo, 2008). The concept of valuable leisure (Cuenca, 2015, Kleiber, Bayón, Cuenca and Monteagudo, 2014), coined by the Institute of Leisure Studies of the University of Deusto, focuses attention on the study of leisure experiences that, being highly significant, manage to transform or improve in somehow its protagonist. Thus, the potential of leisure is highlighted to boost individual and/or collective human development processes through the multiple benefits that it offers (Cuenca, 2015; Kleiber, Walker and Mannell, 2011; Nussbaum, 2012; Porter, Iwasaki and Shank, 2010, Valdemoros, Ponce de León and Gradaille, 2016). A representative sample of 1253 young people (48\% girls and 52\% boys) attending post-compulsory secondary education in public and private centers in Spain completed an ad hoc questionnaire structured in 7 large blocks, such as, leisure and free time, family and school life, health and quality of life, the labor market and entrepreneurship, as well as sociodemographic variables. The sample is representative with an error of $2.3 \%$ and a confidence level of $95 \%$. A quantitative methodological design and simple random sampling have been used. The sections that have been analyzed for this article are those dedicated to leisure and spare time, mainly focusing on the following variables: most significant leisure activities (independent variable), leisure benefits (dependent variable), the organization of the activities, spontaneous, organized or both (independent variable), and spaces for the most significant juvenile leisure (independent variable).

There are three categories of benefits that young Spanish population obtains from their significant leisure: emotional, social and cognitive. For this group, the most significant leisure is an inexhaustible source of emotional benefits. These benefits, associated with the satisfaction of doing what they really like, reveal the intrinsic orientation of these young people towards their leisure, therefore, this practice constitutes a benefit in itself. The emotional benefits have higher scores and their importance does not vary with the variables analyzed. The most significant youth leisure also provides a high dose of social and cognitive benefits.

The most important leisure activities are, in order of significance, physical and sport leisure, cultural activities and parties and celebrations. The physical-sport activities are those that report a greater perception of benefits, mainly physical, emotional, as well as the acquisition of skills and technical skills. Physical-sport leisure is not only the most significant practice, but also the one that gives them a wider range of positive returns. Festivals and celebrations, the third type of preferred activity, provide them with emotional and social benefits. It also draws the attention that cultural activities, chosen by the youth group as one of their priority leisure activities, are a more moderate source of benefits than other less significant activities. Cultural leisure provides, above all, emotional and cognitive benefits, but to a lesser extent than practices such as tourism and hiking or volunteering and associationism. Activities that are a priority for a lower percentage of young people, and yet, for the small percentage who practice them, they are a source of significant emotional, cognitive and especially social benefits. In this sense, it should also be noted that, 
despite the rise of digital leisure among young people, this does play an important role among their most significant practices. Recent studies on digital leisure suggest similar findings (Viñals and Cuenca, 2016).

In all types of benefit, women show lower levels of perception than boys. In addition, the types of benefits perceived by boys and girls are different. The girls claim to obtain technical skills and abilities from their leisure but to a lesser extent than the boys. The same goes for physical benefits, both strongly associated with physical-sport leisure. However, mean scores equated when it comes to emotional benefits, which means that both, boys and girls enjoy a highly satisfying leisure. These results point out the existence of important differences regarding the leisure time of girls and boys (what they find most significant, the time devoted, the frequency or company which they do it with, etc.). However, according to Henderson (2009) it can be concluded that in order to advance the understanding of the role of gender in youthful leisure it is necessary to overcome the usual treatment of this aspect (gender) as an independent variable and to deepen in the factors that are involved in the social construction of gender from more neutral approach.

The results confirm that certain spaces ease the achievement of some types of benefits, in line with the activities they host. Thus, open public spaces congregate, especially, the physical-sport leisure, the popular festivals and being with the friendships. All this explains why this type of space is closely associated with emotional and social benefits. Other closed spaces, such as sports centers, gyms, cinemas, play centers, cultural centers, discotheques or bars, host almost half of the physical and sport leisure activities of young people, as well as a large part of their cultural activities. Hence, in addition to high levels of emotional and social benefits, these spaces are associated with the perception of physical benefits and the acquisition of technical skills and abilities. Finally, private spaces (house, private facilities or markets), which host a large number of youth cultural activities and, to a lesser extent, private parties or celebrations, seem to be the main source of emotional and social benefits. Literature emphasizes that young people prefer and feel more satisfied in leisure spaces that provide them some autonomy. The results of this study reach the same conclusion that the previous ones (Abbot-Champan and Robertson, 2001) and point out that the satisfaction (emotional benefits) of youth is high in all the spaces, not only in private ones (a priori, especially prone to selfmanagement). It also confirms that being satisfied with the particular closed spaces does not necessarily imply that high levels of other categories of benefits are obtained in them. In fact, this study confirms that the greater perception of benefits occurs in the closed non-own spaces, in which leisure activities are organized, which are precisely the type of leisure that emerges as the main source of benefit.

Although more than half of the activities of leisure especially significant for Spanish youth are not held by any entity ( 6 out of 10 ), the results show that the perception of benefits is greater when the activity is performed in an organized framework. This difference in terms of benefits in favor of organized leisure explains why the physical-sport activities and associationism, which are 
most frequently performed in an organized framework, are also those that provide higher levels of benefits. Furthermore, digital leisure and cultural activities, which is performed to a greater extent in an unorganized way, are those that show lower mean scores in all categories of benefits, apart from the emotional ones. These results coincide with those obtained in previous studies (Codina, Pestana, Castillo and Balaguer, 2016, Parra, Oliva and Antolín, 2009, Ramos, Rivera and Moreno, 2012), which confirm that organized leisure encourages youth due to the many benefits it provides. The scientific literature confirms the interest of the youth to engage in organized leisure practices, thereby facilitating a greater achievement of different kinds of benefits. However, these data must be interpreted with caution, bearing in mind that the research about this topic is focused to organized leisure.

There is no evidence of studies that have reviewed the positive returns of unorganized youth leisure. In this article it is the young people themselves who announce that organized leisure is more fruitful, in terms of benefits, than the one they realize on their own. However, far from discarding the benefits of unorganized leisure in the face of evidence of data, we reaffirm the need to continue studying unorganized leisure to clarify the nature or conditions in which the contribution of unorganized leisure to development is optimized youth.

\section{INTRODUCCIÓN}

Entender el ocio juvenil en clave experiencial implica superar nociones de ocio que asumen éste como sinónimo de actividad o tiempo libre, para otorgar relevancia a la calidad de las experiencias que este fenómeno desencadena. Este enfoque exige reconocer que no todas las experiencias y, por ende, todas las manifestaciones del ocio juvenil contribuyen de manera idéntica al desarrollo personal y social (Cuenca, 2015; Monteagudo, 2008).

El concepto de ocio valioso (Cuenca, 2015; Kleiber, Bayón, Cuenca y Monteagudo, 2014), acuñado por el Instituto de Estudios de Ocio de la Universidad de Deusto, permite poner el foco de atención en el estudio de aquellas experiencias (o estilos) de ocio que, siendo altamente significativas, mejoran en algún sentido a su protagonista. Con este concepto se quiere resaltar el potencial del ocio para dinamizar procesos individuales y/o colectivos de desarrollo humano a través de los múltiples beneficios que propicia (Cuenca, 2015; Kleiber, Walker y Mannell, 2011; Nussbaum, 2012; Porter, Iwasaki y Shank, 2010; Valdemoros, Ponce de León y Gradaille, 2016). Si esto es así, parece legítimo el interés científico y social que el ocio juvenil despierta, pues garantizar el cultivo de un ocio positivo, enriquecedor y saludable desde la infancia y juventud, es apostar por el desarrollo juvenil y su continuidad en etapas posteriores de la vida. 


\section{BENEFICIOS DEL OCIO JUVENIL}

El estudio de los beneficios del ocio ha sido abordado como antecedente, explorando las expectativas sobre los retornos positivos que se esperan obtener del ocio (Barnett, 2013; Gibson y Chang, 2012), o como consecuencia, a partir de sus efectos (Barnett, 2013; Codina, Pestana, Castillo y Balaguer, 2016; Sibthorp, Bialeschki, Morgan y Browne, 2013). Durante los últimos años, la literatura especializada se ha decantado por el uso del término resultados (outcomes) del ocio para destacar los efectos tanto positivos como negativos que el ocio puede promover (Kleiber, Walker y Mannel, 2011). Ante esta diversidad de posibilidades, conviene remarcar que este artículo centra la atención únicamente en los efectos positivos del ocio, es decir, en sus beneficios, considerando estos no desde las expectativas, sino en cuanto resultado obtenido del ocio juvenil.

Habitualmente, se entiende por beneficio "un cambio que se percibe como ventajoso, una mejora en algún aspecto que puede favorecer a la persona, grupo, sociedad o cualquier otra entidad" (Driver, Brown y Peterson, 1991, p. 4). Más tarde, Driver y Bruns (1999) avanzaron una triple acepción de este concepto que contempla todas sus vertientes; en primer lugar, como mejora de un estado o condición de una persona o colectivo; en segundo lugar, como prevención de una condición no deseada o el mantenimiento de una condición ya existente y deseada evitando la aparición de una condición no deseable; y, finalmente, como experiencia psicológica satisfactoria. La importancia de esta nueva definición radica en el reconocimiento del valor instrumental y final del ocio (Monteagudo, 2004). En ella se subraya la capacidad del ocio para ser un fin en sí mismo (valor final), fuente primera de satisfacción que no necesita de otros argumentos para justificar la acción, al tiempo que puede actuar como instrumento para el logro de otros objetivos (educativos, sociales, económicos, de salud...) (valor instrumental).

Aunque aún persiste en la literatura una tendencia a justificar la importancia del ocio juvenil, atendiendo únicamente a su naturaleza exotélica o instrumental, cada vez son más los enfoques que subrayan la naturaleza dual del ocio (final e instrumental). Larson (2000) insiste, por ejemplo, en la importancia de que las prácticas de ocio juveniles entusiasmen a este colectivo (valor final del ocio) al tiempo que promueven el desarrollo de habilidades necesarias para sus vidas (valor instrumental). Aún más, algunas investigaciones destacan el papel central de la satisfacción como condición "sine qua non" para la consecución de otros beneficios a través del ocio (Driver y Bruns, 1999; Monteagudo, Ahedo, Richter y Doistua, 2014).

Sibthorp, Bialeschki, Morgan y Browne (2013) estudian los beneficios que los campamentos de verano tienen en la población joven mediante una taxonomía de 11 tipos de beneficios: habilidades sociales, conductas promotoras 
del funcionamiento familiar, responsabilidad, independencia, habilidades de trabajo en grupo, competencia percibida, deseo de exploración, gusto por la Naturaleza, resolución de problemas, conexión con la vida del campamento y bienestar espiritual. Por su parte, Barnett (2013), en su estudio sobre el impacto de la personalidad en las expectativas que las y los jóvenes tienen sobre los retornos de su ocio, emplea una tipología de beneficios de 5 categorías: deseo de nuevas experiencias, deseo de retos y desarrollo de nuevas habilidades, deseo de refuerzos intrínsecos (satisfacción), deseo de relaciones sociales y, finalmente, deseo de ser una persona activa. Larson, Jarrett, Hansen, Pearse, Sullivan, Walker, Watkins y Wood (2004) destacan como consecuencias positivas de la participación de chicos y chicas adolescentes en programas de actividades extraescolares el desarrollo de la iniciativa personal, el fomento de la motivación intrínseca, la adquisición de habilidades sociales, el respeto a la diversidad y el cultivo de la responsabilidad.

\section{APORTACIONES DEL OCIO Y SUS BENEFICIOS AL DESARROLLO JUVENIL: FACTORES DETERMINANTES}

La revisión de estos y otros estudios que forman parte de la abundante literatura existente sobre los beneficios del ocio joven (Chawla, 2015; Doistua y Ried, 2016; Fraguela, Varela y Sanz, 2016; Gardner, Roth y Brooks-Gunn, 2008; Lazcano, Ortega y Baptista, 2015; Mahoney, Larson y Eccles, 2005; Oliva, Pertegal, Antolín, Reina y Ríos, 2011) permiten destacar dos cuestiones fundamentales. En primer lugar, se confirma que la mayoría de las investigaciones realizadas sobre el tema tienen como telón de fondo la teoría del desarrollo positivo juvenil (Damon, 2004) y el modelo de competencia, frente al modelo de déficit que inspiró buena parte de las iniciativas de ocio juvenil institucionales durante las últimas décadas del siglo XX. Desde este enfoque, la juventud ya no es un problema sino un recurso a desarrollar. De ahí que los programas de ocio que deseen contribuir al desarrollo juvenil deben promover una serie de beneficios que permitan a las y los jóvenes transitar con éxito por esta etapa vital y alcanzar una madurez ajustada (Parra, Oliva, Antolín, 2009). En segundo lugar, se evidencia que tanto los beneficios del ocio como el logro del desarrollo juvenil están mediados por variables diversas que han de considerarse al diseñar programas orientados a la promoción del ocio juvenil. Entre estas variables merece la pena destacar el carácter organizado o no organizado del ocio juvenil, los espacios en los que tiene lugar y el género.

- Ocio organizado versus no organizado

Desde el modelo de competencia que inspira la comprensión de la etapa juvenil como un periodo para el cultivo de capacidades (Damon, 2004; Parra, Oliva y Antolín, 2009), se defiende la importancia de que las y los jóvenes par- 
ticipen en programas de ocio organizado. Estos programas se llevan a cabo desde una institución y bajo la supervisión de una persona adulta y formada (Zeijl, Du Bois-Reymond y Te Poel, 2001) que orienta su intervención hacia la construcción de habilidades asociadas al desarrollo juvenil (Eccles y Gootman, 2002; Roth y Brooks-Gunn, 2003). Dichos programas disponen de unos objetivos claros que se pretenden alcanzar a través de actividades planificadas. Investigaciones previas demuestran que las y los jóvenes que participan en actividades de ocio organizadas perciben mayores beneficios que quienes practican su ocio de forma libre (Ahedo y Macua, 2016). Este tipo de ocio juvenil está asociado a un mayor compromiso social (Gardner, Roth y Brooks-Gunn, 2008), un mejor ajuste emocional (Broh, 2002) y una mayor disponibilidad de habilidades sociales (Devine y Parr, 2008). Estudios longitudinales confirman, además, los efectos positivos de estos programas, incluso una vez concluida la participación (Gardner, Roth y Brooks-Gunn, 2008). Los datos disponibles sobre el ocio juvenil revelan, sin embargo, que la mayoría de las prácticas de ocio joven son actividades no estructuradas (Mahoney, Larson y Eccles, 2005; Ramos, Rivera y Moreno, 2012).

- Espacios para el ocio juvenil

Los espacios para el ocio juvenil son componentes esenciales del mismo por su capacidad de modular las experiencias que acogen, así como los beneficios asociados a dichas experiencias. La literatura identifica una serie de rasgos que deberían compartir estos espacios cuando el objetivo a alcanzar es el desarrollo juvenil; entre otros, el acceso y uso de espacios física y psicológicamente seguros en los que sea posible la apropiación y construcción colectiva de significados. El carácter público o privado de estos espacios o la mayor o menor normativización adulta que prevalece en ellos (Tejerina, Carbajo y Martínez 2012) son también variables relevantes a la hora de entender el ocio juvenil. Las personas jóvenes buscan espacios que les proporcionen cierta distancia del mundo adulto (Abbot-Champan y Robertson, 2001) y obtienen más satisfacción en espacios en los que disfrutan de mayor autonomía y posibilidades de autogestión (Ortega, Lazcano y Baptista, 2015).

- Género

El género es una de las variables sociodemográficas que más incide en la configuración de las experiencias de ocio juvenil y en sus beneficios. Como variable independiente, el género parece modular las decisiones que la población toma respecto a su ocio: las modalidades que práctica, el tiempo dedicado, los motivos que respaldan la práctica o el tipo de barreras que percibe para continuar practicando (Henderson, 2009). Diferencias que se constatan ya durante 
la infancia y juventud y que, en muchos casos, persisten en etapas tardías de la vida (Kleiber, Bayón, Cuenca y Monteagudo, 2014). Investigaciones recientes (Barnett, 2013) revelan que las chicas son menos proclives que los chicos a buscar la adquisición de destrezas o habilidades, atribuyen menos importancia al disfrute y están menos predispuestas a permanecer activas a través de su ocio.

\section{MÉTODO}

\section{Participantes}

Este trabajo se enmarca dentro del proyecto coordinado "De los tiempos educativos a los tiempos sociales: la construcción cotidiana de la condición juvenil en una sociedad de redes", cuyo universo es la población joven española (16-18 años). Se ha utilizado un diseño metodológico cuantitativo y un muestreo aleatorio simple entre las y los jóvenes que cursaban Educación Secundaria Postobligatoria (Bachiller, Formación Profesional de Grado Medio y Programas de Capacitación Profesional Inicial) en centros públicos y privados del territorio español, tratando de mantener una afijación proporcional en cada una de las seis áreas en las que se dividió el territorio español: Noreste: Cataluña, Baleares y Aragón (excepto Teruel incluida en el área Centro); Levante: Comunidad Valenciana, Murcia y Albacete; Sur: Andalucía, Islas Canarias, Ceuta y Melilla; Centro: Madrid, Castilla-La Mancha, Castilla-León, Cáceres y Teruel (excepto Albacete, León, Palencia y Burgos incluidas en las áreas Norte y Noroeste); Noroeste: Galicia, Asturias y León; Norte: Cantabria, País Vasco, La Rioja, Navarra, Burgos y Palencia.

La muestra está compuesta por un total de 1253 jóvenes (48\% chicas y $52 \%$ chicos). Se trata de una muestra representativa con un error del 2,3\% y un nivel de confianza del 95\%.

\section{Instrumento}

Se elaboró un cuestionario ad hoc, orientado a dar respuesta a los objetivos planteados para el conjunto de la investigación coordinada. El cuestionario abarcaba un amplio espectro de cuestiones centradas en 7 grandes bloques: ocio y tiempo libre, vida familiar y en el centro escolar, salud y calidad de vida así como mercado laboral y emprendimiento, además de las variables sociodemográficas. Este artículo se centra únicamente en el apartado dedicado al ocio y tiempo libre y, fundamentalmente, en las siguientes variables:

Actividades de ocio más significativas (variable independiente): cada joven debía seleccionar, entre todas las actividades de ocio practicadas, las tres más 
significativas; es decir, aquellas que consideraba especialmente importantes y de las que no le gustaría prescindir. Aunque era una pregunta abierta, disponía de una ficha de apoyo en la que se presentaban distintas actividades de ocio agrupadas en 8 categorías: actividades culturales (escuchar música, ver televisión o películas, ir al cine, teatro, museos o exposiciones, asistir a conciertos o espectáculos deportivos, realizar alguna actividad artística...), ocio digital (escribir un blog o crear páginas web, participar en chats o redes sociales, videojuegos, juegos de azar online..), actividad físico-deportiva (individuales, grupales, de cooperación u oposición...), turismo o excursionismo, fiestas y celebraciones, juegos sin ordenador, hobbies y asociacionismo/voluntariado.

Beneficios del ocio juvenil más significativo (variable dependiente). Mediante una escala tipo Likert de 5 puntos que iba desde 1 . Nada de acuerdo hasta 5 . Muy de acuerdo, se les solicitaba su grado de acuerdo con un total de 5 afirmaciones, cada una asociada a la percepción de un tipo de beneficio: a) Estoy más en forma, controlo mejor mis movimientos, mantengo o mejoro mi condición física, etc. (Beneficio físico); b) Me siento más satisfecho/a, disfruto haciéndolo, me divierte, etc. (Beneficio emocional); c) Soy más creativo/a, adquiero conocimientos, aprendo cosas, amplio mi mundo, etc. (Beneficio cognitivo); d) Desarrollo nuevas destrezas manuales y adquiero o perfecciono habilidades técnicas (Adquisición de destrezas y habilidades técnicas); e) Hago cosas diferentes con más gente, me siento parte de un grupo, me ayuda a relacionarme mejor con los demás, etc. (Beneficio social).

Carácter organizado, espontáneo o ambos del ocio juvenil más significativo (variable independiente). Mediante una pregunta simple, de respuesta si/no, debían indicar si realizaban sus prácticas de ocio seleccionadas en el marco de una organización, por su cuenta o de ambas maneras.

Espacios para el ocio juvenil más significativo (variable independiente). Para el análisis de los espacios en los que tenían lugar las actividades de ocio seleccionadas se recodificaron las 8 opciones de respuesta proporcionadas en tres tipos de espacios: espacios abiertos (espacios naturales y espacios públicos abiertos como la calle, parques o plazas); espacios cerrados particulares (locales privados, cedidos a alquilados que se comparten con gente de la misma edad; la casa, urbanizaciones u otras residencias privadas; asociaciones, clubes, sociedades o peñas donde hay gente de distintas edades); espacios cerrados ajenos (espacios municipales como polideportivos, centros culturales, ludotecas; las instalaciones del centro escolar; espacios de ocio como centros comerciales, zonas de bares, cines y discotecas). 


\section{Procedimiento}

La recogida de información se realizó de forma presencial en centros escolares de las diferentes Comunidades Autónomas del contexto español. Una vez contactados los centros educativos, se solicitó a las y los directores su colaboración y se seleccionaron aleatoriamente las clases en las que se aplicaría el cuestionario. El trabajo de campo se realizó de octubre de 2014 a febrero de 2015. Dos investigadores se desplazaron a cada centro educativo donde se administró el cuestionario en un aula de forma grupal. La participación era voluntaria y anónima.

\section{Análisis de datos}

Para el análisis de los datos se utilizó el software SPSS (v. 21). El análisis descriptivo del ocio juvenil, su organización, así como los lugares en los que se desarrolla se realiza mediante frecuencias y porcentajes. El análisis de los beneficios se presenta mediante las correspondientes medias y desviaciones típicas. Por último, la asociación de los beneficios con las variables género, edad, estudios, organización de las actividades y lugar de realización se lleva a cabo mediante la prueba U de Mann-Whitney.

\section{Resultados}

Esta sección presenta los resultados obtenidos en relación con la percepción de beneficios derivados de las prácticas de ocio de las y los jóvenes españoles (16-18 años). En los siguientes apartados se abordará la relación entre estos beneficios y el género, la modalidad de ocio practicada, el carácter organizado o no organizado de la actividad y el lugar en el que se realiza. Los datos obtenidos no muestran diferencias significativas entre percepción de beneficios y la edad o el tipo de estudios. Por lo que no se incidirá nuevamente en el estudio de estas variables.

\section{BENEFICIOS DEL OCIO JUVENIL EN ESPAÑA}

La población joven española percibe principalmente tres tipos de beneficios asociados a sus prácticas de ocio más significativas: beneficios emocionales (media de 4,38 sobre 5 ), sociales $(3,48)$ y cognitivos $(3,46)$.

Esta relevancia se mantiene e incluso se hace más evidente, considerando de manera independiente el orden de importancia de las prácticas de ocio juvenil más significativas. Las puntuaciones medias de los beneficios emocionales son notablemente más altas que las del resto en las tres actividades (Tabla 1). 
Tabla 1. Puntuaciones medias de percepción de beneficios de las tres actividades de ocio juvenil más significativas en conjunto y, según importancia de cada actividad

\begin{tabular}{|c|c|c|c|c|c|c|c|c|}
\hline & \multicolumn{2}{|c|}{$\begin{array}{l}\text { Conjunto de } \\
\text { actividades }\end{array}$} & \multicolumn{2}{|c|}{$\begin{array}{l}1^{\mathrm{a}} \text { Actividad } \\
\text { de ocio }\end{array}$} & \multicolumn{2}{|c|}{$\begin{array}{l}2^{\mathrm{a}} \text { Actividad } \\
\text { de ocio }\end{array}$} & \multicolumn{2}{|c|}{$\begin{array}{c}3^{\text {a }} \text { Actividad } \\
\text { de ocio }\end{array}$} \\
\hline & Media & $D E$ & Media & $D E$ & Media & $D E$ & Media & $D E$ \\
\hline Beneficios físicos & 2,69 & 1,60 & 3,00 & 1,66 & 2,66 & 1,59 & 2,39 & 1,48 \\
\hline Beneficios emocionales & 4,38 & 0,92 & 4,54 & 0,82 & 4,39 & 0,90 & 4,22 & 1,00 \\
\hline Beneficios cognitivos & 3,46 & 1,28 & 3,56 & 1,30 & 3,44 & 1,27 & 3,36 & 1,27 \\
\hline $\begin{array}{l}\text { Adquisición destrezas y } \\
\text { habilidades técnicas }\end{array}$ & 2,85 & 1,51 & 3,13 & 1,56 & 2,80 & 1,48 & 2,62 & 1,45 \\
\hline Beneficios sociales & 3,48 & 1,48 & 3,84 & 1,41 & 3,42 & 1,47 & 3,16 & 1,49 \\
\hline Beneficio medio & 3,40 & 0,93 & 3,63 & 0,94 & 3,37 & 0,91 & 3,18 & 0,89 \\
\hline
\end{tabular}

Tabla 2. Puntuaciones medias de percepción de beneficios del ocio juvenil más significativo, según género

\begin{tabular}{|c|c|c|c|c|c|c|c|}
\hline & \multicolumn{3}{|c|}{ Género } & \multirow{2}{*}{\multicolumn{4}{|c|}{ Estadísticos de contraste }} \\
\hline & \multirow{2}{*}{\begin{tabular}{|l} 
Mujer \\
Media
\end{tabular}} & \multirow{2}{*}{$\begin{array}{c}\text { Hombre } \\
\text { Media }\end{array}$} & \multirow{2}{*}{\begin{tabular}{|c|} 
Total \\
Media
\end{tabular}} & & & & \\
\hline & & & & $\begin{array}{l}\text { U de Mann- } \\
\text { Whitney }\end{array}$ & $\begin{array}{c}W \text { de } \\
\text { Wilcoxon }\end{array}$ & $z$ & $\begin{array}{c}\text { Sig. asintót. } \\
\text { (bilateral) }\end{array}$ \\
\hline $\begin{array}{l}\text { Beneficios } \\
\text { físicos }\end{array}$ & 2,43 & 2,93 & 2,69 & 910801 & 1915454,000 & $-8,390$ & 0,000 \\
\hline $\begin{array}{l}\text { Beneficios } \\
\text { emocionales }\end{array}$ & 4,37 & 4,39 & 4,38 & 1147531,5 & 2228716,500 & $-1,158$ & 0,247 \\
\hline $\begin{array}{l}\text { Beneficios } \\
\text { cognitivos }\end{array}$ & 3,36 & 3,55 & 3,46 & 1053603 & 2117214,000 & $-3,829$ & 0,000 \\
\hline $\begin{array}{l}\text { Adquisición } \\
\text { destrezas y } \\
\text { habilidades } \\
\text { técnicas }\end{array}$ & 2,50 & 3,18 & 2,85 & 832334,5 & 1861229,500 & $-12,350$ & 0,000 \\
\hline $\begin{array}{l}\text { Beneficios } \\
\text { sociales }\end{array}$ & 3,33 & 3,62 & 3,48 & 1013086 & 2059267,000 & $-5,110$ & 0,000 \\
\hline $\begin{array}{l}\text { Beneficio } \\
\text { medio }\end{array}$ & 3,23 & 3,56 & 3,40 & 980849 & 2124677,000 & $-9,839$ & 0,000 \\
\hline
\end{tabular}


Este dato es importante porque subraya el interés intrínseco de las y los jóvenes en las actividades indicadas. Quienes perciben este tipo de beneficio afirman que su satisfacción deriva de hacer aquello que les gusta y satisface. En este orden, los beneficios sociales y cognitivos son también importantes.

\section{LOS BENEFICIOS DEL OCIO JUVENIL, SEGÚN GÉNERO}

El género emerge como una variable relevante a la hora de analizar los beneficios que la población joven obtiene de su ocio (Tabla 2). Las chicas presentan medias inferiores que las de los chicos en todos los tipos de beneficios, excepto en el caso de los emocionales.

Estas diferencias resultan estadísticamente significativas y se hacen especialmente notorias cuando se trata de la adquisición de destrezas y habilidades técnicas (diferencia de 0,68 puntos a favor de los chicos) y de beneficios físicos (diferencia de 0,5 puntos a favor de los chicos).

\section{RELACIÓN DEL TIPO DE ACTIVIDAD DE OCIO CON LOS BENEFICIOS PERCIBIDOS}

Consideradas en su conjunto, las tres actividades que el colectivo joven indica como sus prácticas de ocio más significativas son las culturales $(25,4 \%)$, las físico-deportivas $(21,45)$ y las fiestas y celebraciones $(14,2 \%)$ (Tabla 3). El $22 \%$ de las y los jóvenes opta por la categoría "Otras actividades", en la que destacan las respuestas que consideran "estar con las amistades" como actividad de ocio prioritaria.

El análisis individual de cada una de las tres prácticas de ocio identificadas como más significativas revela que la actividad principal es la físico-deportiva $(29,7 \%)$; le siguen las prácticas culturales $(20,9 \%)$ y las fiestas y celebraciones (17\%). A mayor distancia, destaca el ocio digital como actividad más relevante para el 9,5\% (Tabla 3). Las actividades culturales adquieren protagonismo como segundas prácticas de ocio prioritarias $(27,1 \%)$ y el ocio digital se equipara en importancia al ocio físico-deportivo cuando se trata de las terceras prácticas de ocio más significativas ( $12,3 \%$ y $13,6 \%$, respectivamente) (Tabla 3).

El cruce del tipo de práctica con la naturaleza de los beneficios percibidos permite detectar los beneficios que las y los jóvenes identifican como consecuencia de sus prácticas de ocio preferidas (Tabla 4). Los beneficios físicos están asociados preferentemente a las actividades físico-deportivas, mientras que apenas se relacionan con el ocio digital. Los beneficios emocionales son los más habituales en todas las actividades pero su percepción es especialmente eleva- 
Tabla 3. Práctica de las actividades de ocio juvenil más significativas en conjunto y según importancia de cada actividad ( $\mathrm{n}$ actividades=3759)

\begin{tabular}{|c|c|c|c|c|c|c|c|c|}
\hline & \multicolumn{2}{|c|}{$\begin{array}{l}\text { Conjunto de } \\
\text { actividades }\end{array}$} & \multicolumn{2}{|c|}{$\begin{array}{l}1^{\mathrm{a}} \text { Actividad } \\
\text { de ocio }\end{array}$} & \multicolumn{2}{|c|}{$\begin{array}{l}2^{\text {a }} \text { Actividad } \\
\text { de ocio }\end{array}$} & \multicolumn{2}{|c|}{$\begin{array}{c}3^{\mathrm{a}} \text { Actividad } \\
\text { de ocio }\end{array}$} \\
\hline & $\mathrm{N}$ & $\%$ & $\mathrm{~N}$ & $\%$ & $\mathbf{N}$ & $\%$ & $\mathrm{~N}$ & $\%$ \\
\hline Actividades culturales & 955 & $25,4 \%$ & 262 & $20,9 \%$ & 340 & $27,1 \%$ & 353 & $28,2 \%$ \\
\hline Ocio Digital & 407 & $10,8 \%$ & 119 & $9,5 \%$ & 134 & $10,7 \%$ & 154 & $12,3 \%$ \\
\hline $\begin{array}{l}\text { Actividad físico- } \\
\text { deportiva }\end{array}$ & 804 & $21,4 \%$ & 372 & $29,7 \%$ & 261 & $20,8 \%$ & 171 & $13,6 \%$ \\
\hline $\begin{array}{l}\text { Turismo o } \\
\text { Excursionismo }\end{array}$ & 75 & $2,0 \%$ & 13 & $1,0 \%$ & 21 & $1,7 \%$ & 41 & $3,3 \%$ \\
\hline Fiestas y Celebraciones & 534 & $14,2 \%$ & 213 & $17,0 \%$ & 163 & $13,0 \%$ & 158 & $12,6 \%$ \\
\hline Juegos & 19 & $0,5 \%$ & 5 & $0,4 \%$ & 4 & $0,3 \%$ & 10 & $0,8 \%$ \\
\hline Hobbies & 95 & $2,5 \%$ & 9 & $0,7 \%$ & 37 & $3,0 \%$ & 49 & $3,9 \%$ \\
\hline $\begin{array}{l}\text { Asociacionismo- } \\
\text { Voluntariado }\end{array}$ & 18 & $0,5 \%$ & 6 & $0,5 \%$ & 5 & $0,4 \%$ & 7 & $0,6 \%$ \\
\hline Otros - Varios & 852 & $22,7 \%$ & 254 & $20,3 \%$ & 288 & $23,0 \%$ & 310 & $24,7 \%$ \\
\hline
\end{tabular}

da en las actividades físico-deportivas (media de 4,59 sobre 5), en fiestas y celebraciones y en el ámbito del asociacionismo y voluntariado (medias de 4,57, en ambos casos) así como en el turismo y excursionismo (media de 4,53). Por contraste, en esta misma categoría de beneficios, el ocio digital es la actividad que muestra una media más baja (media de 4,08). Por su parte, los beneficios cognitivos derivan de prácticas como el asociacionismo y voluntariado $(4,54)$, el turismo o excursionismo $(4,05)$, así como de las actividades culturales $(3,75)$. La adquisición de destrezas y habilidades técnicas se asocia principalmente con la actividad físico-deportiva $(3,98)$, mientras que apenas mantiene relación con el ámbito de las fiestas y celebraciones $(2,05)$. Finalmente, la contribución del ocio al mundo social de las y los jóvenes adopta su máxima expresión en el mundo del asociacionismo y voluntariado, en las fiestas y celebraciones y en la realización de actividades físico-deportivas (4,79, 4,47 y 4,02, respectivamente).

\section{LA ORGANIZACIÓN DEL OCIO JUVENIL Y SU RELACIÓN CON LA PERCEPCIÓN DE BENEFICIOS}

El carácter organizado o espontáneo del ocio joven es otra variable a tener en cuenta en el análisis de la percepción de beneficios. El 60,9\% de las actividades 
Tabla 4. Puntuaciones medias de percepción de beneficios de las tres actividades de ocio juvenil más significativas en conjunto, según tipo de actividad

\begin{tabular}{|c|c|c|c|c|c|c|}
\hline & $\begin{array}{c}\text { Beneficios } \\
\text { físicos }\end{array}$ & $\begin{array}{c}\text { Beneficios } \\
\text { emocionales }\end{array}$ & \begin{tabular}{|l|} 
Beneficios \\
cognitivos
\end{tabular} & $\begin{array}{c}\text { Adquisición } \\
\text { destrezas y } \\
\text { habilidades } \\
\text { técnicas }\end{array}$ & $\begin{array}{l}\text { Beneficios } \\
\text { sociales }\end{array}$ & $\begin{array}{l}\text { Beneficio } \\
\text { medio }\end{array}$ \\
\hline $\begin{array}{l}\text { Actividades } \\
\text { culturales }\end{array}$ & 1,80 & 4,25 & 3,75 & 2,41 & 2,45 & 2,98 \\
\hline Ocio Digital & 1,72 & 4,08 & 3,51 & 2,80 & 3,14 & 3,09 \\
\hline $\begin{array}{l}\text { Actividad físico- } \\
\text { deportiva }\end{array}$ & 4,50 & 4,59 & 3,52 & 3,98 & 4,02 & 4,13 \\
\hline $\begin{array}{l}\text { Turismo o } \\
\text { Excursionismo }\end{array}$ & 3,44 & 4,53 & 4,05 & 2,82 & 3,87 & 3,78 \\
\hline $\begin{array}{l}\text { Fiestas y } \\
\text { Celebraciones }\end{array}$ & 2,13 & 4,57 & 2,89 & 2,05 & 4,47 & 3,27 \\
\hline Juegos & 2,13 & 4,13 & 2,94 & 3,13 & 3,75 & 3,26 \\
\hline Hobbies & 2,46 & 4,32 & 2,83 & 2,36 & 3,10 & 3,04 \\
\hline $\begin{array}{l}\text { Asociacionismo- } \\
\text { Voluntariado }\end{array}$ & 2,77 & 4,57 & 4,54 & 3,08 & 4,79 & 4,01 \\
\hline Otros - Varios & 2,72 & 4,36 & 3,39 & 2,87 & 3,65 & 3,43 \\
\hline Total & 2,69 & 4,38 & 3,46 & 2,85 & 3,48 & 3,40 \\
\hline \multicolumn{7}{|c|}{ Estadísticos de contraste } \\
\hline Chi-cuadrado & 1209,285 & 109,732 & 175,212 & 554,594 & 688,606 & 673,526 \\
\hline gl & 7,000 & 7,000 & 7,000 & 7,000 & 7,000 & 7,000 \\
\hline Sig. Asintót. & 0,000 & 0,000 & 0,000 & 0,000 & 0,000 & 0,000 \\
\hline
\end{tabular}

del ocio juvenil se realiza de forma libre, al margen de cualquier entidad (Tabla 5). Este porcentaje es especialmente elevado en el caso del ocio digital (84\%) o las actividades culturales $(75,7 \%)$, pero se reduce considerablemente en actividades como asociacionismo/voluntariado $(11,1 \%)$ y el ocio físico-deportivo $(34,5 \%)$. Se trata en ambos casos de actividades que se llevan a cabo, sobre todo, en el marco de una organización (55,6\% y 36,6\%, respectivamente).

Los datos confirman el impacto positivo del ocio organizado en la percepción de beneficios. La puntuación media de beneficios alcanza un 3,85 sobre 5 cuando se trata de un ocio organizado, mientras que desciende hasta el 3,19 en 
Tabla 5. Práctica de las actividades de ocio juvenil más significativas en conjunto y según su carácter organizado o espontáneo (n actividades $=3645$ )

\begin{tabular}{|c|c|c|c|c|c|c|}
\hline & \multicolumn{6}{|c|}{ Organización } \\
\hline & \multicolumn{2}{|c|}{$\begin{array}{l}\text { De forma libre, } \\
\text { por mi cuenta }\end{array}$} & \multicolumn{2}{|c|}{$\begin{array}{l}\text { De forma } \\
\text { organizada, en } \\
\text { una asociación, } \\
\text { club, etc. }\end{array}$} & \multicolumn{2}{|c|}{ Ambas } \\
\hline & $\mathrm{N}$ & $\%$ & $\mathrm{~N}$ & $\%$ & $\mathrm{~N}$ & $\%$ \\
\hline Actividades culturales & 723 & $75,7 \%$ & 53 & $5,5 \%$ & 109 & $11,4 \%$ \\
\hline Ocio Digital & 342 & $84,0 \%$ & 11 & $2,7 \%$ & 25 & $6,1 \%$ \\
\hline Actividad físico-deportiva & 277 & $34,5 \%$ & 294 & $36,6 \%$ & 176 & $21,9 \%$ \\
\hline Turismo o Excursionismo & 36 & $48,0 \%$ & 16 & $21,3 \%$ & 16 & $21,3 \%$ \\
\hline Fiestas y Celebraciones & 322 & $60,3 \%$ & 100 & $18,7 \%$ & 75 & $14,0 \%$ \\
\hline Juegos & 11 & $57,9 \%$ & 2 & $10,5 \%$ & 5 & $26,3 \%$ \\
\hline Hobbies & 68 & $71,6 \%$ & 6 & $6,3 \%$ & 10 & $10,5 \%$ \\
\hline $\begin{array}{l}\text { Asociacionismo- } \\
\text { Voluntariado }\end{array}$ & 2 & $11,1 \%$ & 10 & $55,6 \%$ & 6 & $33,3 \%$ \\
\hline Otros - Varios & 440 & $59,6 \%$ & 124 & $16,8 \%$ & 92 & $12,5 \%$ \\
\hline Total & 2221 & $60,9 \%$ & 616 & $16,9 \%$ & 514 & $14,1 \%$ \\
\hline
\end{tabular}

el caso del ocio que las y los jóvenes realizan por su cuenta (Tabla 6). Esta diferencia a favor del ocio organizado se confirma en todos los tipos de beneficios, excepto en los emocionales. El único tipo de beneficio que se obtiene a través del ocio organizado y no organizado.

\section{LOS ESPACIOS DE OCIO JUVENIL Y SU CONEXIÓN CON LOS BENEFICIOS PERCIBIDOS}

Con el fin de conocer la naturaleza de los espacios en los que transcurre el ocio juvenil en España, se presentan los resultados obtenidos en las tres categorías de espacios consideradas: abiertos, cerrados particulares y cerrados ajenos (Tabla 7).

Los espacios públicos abiertos acogen principalmente el ocio físico-deportivo $(23,8 \%)$ que se realiza en la calle, en lugares públicos, en muchos casos, habilitados para ello como pueden ser plazas o parques. Estos espacios son también principales aliados de fiestas y celebraciones (23,7\%). Los espacios cerrados 
Tabla 6. Puntuaciones medias de los beneficios percibidos de las tres actividades de ocio juvenil más significativas en conjunto, según su carácter organizado o espontáneo (n actividades=3759)

\begin{tabular}{|c|c|c|c|c|c|c|}
\hline & $\begin{array}{c}\text { Beneficios } \\
\text { físicos } \\
\text { Media }\end{array}$ & $\begin{array}{c}\text { Beneficios } \\
\text { emocionales } \\
\text { Media }\end{array}$ & $\begin{array}{c}\text { Beneficios } \\
\text { cognitivos } \\
\text { Media }\end{array}$ & \begin{tabular}{|c|} 
Adquisición \\
destrezas y \\
habilidades \\
técnicas \\
Media \\
\end{tabular} & $\begin{array}{c}\text { Beneficios } \\
\text { sociales } \\
\text { Media }\end{array}$ & $\begin{array}{c}\text { Total } \\
\text { Media }\end{array}$ \\
\hline $\begin{array}{l}\text { De forma libre, } \\
\text { por mi cuenta } \\
\text { (no organizado) }\end{array}$ & 2,29 & 4,34 & 3,41 & 2,55 & 3,15 & 3,19 \\
\hline $\begin{array}{l}\text { De forma } \\
\text { organizada, en } \\
\text { una asociación, } \\
\text { club, etc. }\end{array}$ & 3,50 & 4,52 & 3,50 & 3,46 & 4,24 & 3,85 \\
\hline Ambas & 3,38 & 4,50 & 3,61 & 3,35 & 3,99 & 3,77 \\
\hline $\mathrm{Ns} / \mathrm{Nc}$ & 2,76 & 4,14 & 3,44 & 2,99 & 3,42 & 3,43 \\
\hline Total & 2,69 & 4,38 & 3,46 & 2,85 & 3,48 & 3,40 \\
\hline \multicolumn{7}{|c|}{ Estadísticos de contraste } \\
\hline Chi-cuadrado & 0,000 & 0,000 & 0,009 & 0,000 & 0,000 & 0,000 \\
\hline gl & 2,000 & 2,000 & 2,000 & 2,000 & 2,000 & 2,000 \\
\hline Sig. asintót. & 319,607 & 22,256 & 9,444 & 201,495 & 281,825 & 309,789 \\
\hline
\end{tabular}

propios emergen como contextos idóneos para actividades culturales $(31,1 \%)$ y también para fiestas y celebraciones $(17,4 \%)$. Por su parte los espacios cerrados ajenos son propicios para el ocio físico-deportivo $(47,5 \%)$ y para algunas actividades culturales $(17,9 \%)$. El porcentaje de actividades culturales que tiene lugar en espacios cerrados ajenos es inferior al de las que transcurren en espacios cerrados propios tales como la propia casa o locales particulares, cedidos o alquilados que, en algunas comunidades autónomas reciben el nombre de lonjas. Dichas lonjas son casi siempre espacios auto-gestionados por las y los propios jóvenes y en los que disfrutan de prácticas culturales, así como de fiestas y celebraciones particulares. Finalmente, los espacios cerrados ajenos (polideportivos o instalaciones del centro escolar, entre otros) acogen casi la mitad de las actividades físico-deportivas $(47,5 \%)$ y, en menor medida, algunas actividades culturales $(17,9 \%)$. Dado que se trata de espacios cerrados ajenos bien puede pensarse en cines, centros culturales o ludotecas, como algunos de los espacios que utiliza el colectivo joven para estas actividades culturales. 
Tabla 7. Espacios en los que tiene lugar ocio juvenil más significativo ( $\mathrm{n}$ actividades $=3759$ )

\begin{tabular}{l|c|c|c|c|c|c}
\hline & \multicolumn{2}{|c|}{$\begin{array}{c}\text { Espacios públicos } \\
\text { abiertos }\end{array}$} & $\begin{array}{c}\text { Espacios cerrados } \\
\text { propios }\end{array}$ & \multicolumn{2}{|c}{$\begin{array}{c}\text { Espacios } \\
\text { cerrados ajenos }\end{array}$} \\
\hline & $\mathrm{N}$ & $\%$ & $\mathrm{~N}$ & $\%$ & $\mathrm{~N}$ & $\%$ \\
\hline Actividades culturales & 158 & $13,7 \%$ & 765 & $31,1 \%$ & 142 & $17,9 \%$ \\
\hline Ocio Digital & 71 & $6,2 \%$ & 352 & $14,3 \%$ & 57 & $7,2 \%$ \\
\hline Actividad físico-deportiva & 274 & $23,8 \%$ & 335 & $13,6 \%$ & 377 & $47,5 \%$ \\
\hline Turismo o Excursionismo & 52 & $4,5 \%$ & 13 & $0,5 \%$ & 5 & $0,6 \%$ \\
\hline Fiestas y Celebraciones & 272 & $23,7 \%$ & 427 & $17,4 \%$ & 42 & $5,3 \%$ \\
\hline Juegos & 2 & $0,2 \%$ & 14 & $0,6 \%$ & 4 & $0,5 \%$ \\
\hline Hobbies & 20 & $1,7 \%$ & 74 & $3,0 \%$ & 3 & $0,4 \%$ \\
\hline $\begin{array}{l}\text { Asociacionismo- } \\
\text { Voluntariado }\end{array}$ & 6 & $0,5 \%$ & 11 & $0,4 \%$ & 8 & $1,05 \%$ \\
\hline Otros - Varios & 295 & $25,7 \%$ & 468 & $19,0 \%$ & 156 & $19,6 \%$ \\
\hline
\end{tabular}

La consideración de los beneficios, atendiendo al tipo de espacios indica que los beneficios físicos están asociados a espacios cerrados ajenos $(3,61)$, en los que transcurre buena parte de su ocio físico-deportivo (Tabla 8). A pesar de que los beneficios emocionales son elevados en todos los tipos de espacios, los espacios públicos abiertos son el principal marco para la percepción de estos retornos $(4,54)$. Los beneficios cognitivos son ligeramente más habituales en espacios cerrados ajenos $(3,63)$. La adquisición de destrezas y habilidades técnicas tiene lugar principalmente en espacios cerrados ajenos $(3,55)$. Finalmente, el cultivo del mundo social de las y los jóvenes se lleva a cabo sobre todo en espacios cerrados ajenos $(4,02)$ y en públicos abiertos $(3,88)$. Recordemos que espacios de ocio como centros comerciales, zonas de bares, cines y discotecas están considerados en esta categoría, espacios cerrados ajenos.

\section{DISCUSIÓN Y CONCLUSIONES}

Este estudio sobre el ocio de la población joven española (16-18 años) y los beneficios que este ámbito les reporta centra su atención en aquellas prácticas de ocio que las y los propios jóvenes identifican como significativas, esto es, aquellas que les resultan especialmente importantes y de las cuales no quisieran prescindir. Este enfoque, totalmente en línea con la comprensión del ocio joven en 
Tabla 8. Puntuaciones medias de los beneficios percibidos de las tres actividades de ocio juvenil más significativas, según el tipo de espacio ( $\mathrm{n}$ actividades $=3759$ )

\begin{tabular}{l|c|c|c|c|c|c}
\hline & $\begin{array}{c}\text { Beneficios } \\
\text { físicos } \\
\text { Media }\end{array}$ & $\begin{array}{c}\text { Beneficios } \\
\text { emocionales } \\
\text { Media }\end{array}$ & $\begin{array}{c}\text { Beneficios } \\
\text { cognitivos } \\
\text { Media }\end{array}$ & $\begin{array}{c}\text { Adquisición } \\
\text { destrezas y } \\
\text { habilidades } \\
\text { técnicas } \\
\text { Media }\end{array}$ & $\begin{array}{c}\text { Beneficios } \\
\text { sociales } \\
\text { Media }\end{array}$ & $\begin{array}{c}\text { Beneficio } \\
\text { medio } \\
\text { Media }\end{array}$ \\
\hline $\begin{array}{l}\text { Espacios } \\
\text { públicos } \\
\text { abiertos }\end{array}$ & 2,97 & 4,54 & 3,45 & 2,84 & 3,88 & 3,55 \\
\hline $\begin{array}{l}\text { Espacios } \\
\text { cerrados } \\
\text { particulares }\end{array}$ & 2,36 & 4,36 & 3,43 & 2,66 & 3,39 & 3,27 \\
\hline $\begin{array}{l}\text { Espacios } \\
\text { cerrados ajenos }\end{array}$ & 3,61 & 4,47 & 3,63 & 3,55 & 4,02 & 3,87 \\
\hline
\end{tabular}

Estadísticos de contraste (Sig. Asintót bilateral)

\begin{tabular}{l|c|c|c}
\hline & $\begin{array}{c}\text { Espacios públicos } \\
\text { abiertos }\end{array}$ & $\begin{array}{c}\text { Espacios cerrados } \\
\text { particulares }\end{array}$ & $\begin{array}{c}\text { Espacios cerrados } \\
\text { ajenos }\end{array}$ \\
\hline Beneficios físicos & 0,000 & 0,000 & 0,000 \\
\hline Beneficios emocionales & 0,000 & 0,012 & 0,002 \\
\hline Beneficios cognitivos & 0,662 & 0,094 & 0,000 \\
\hline $\begin{array}{l}\text { Adquisición destrezas } \\
\text { y habilidades técnicas }\end{array}$ & 0,878 & 0,000 & 0,000 \\
\hline Beneficios sociales & 0,000 & 0,000 & 0,000 \\
\hline Beneficio medio & 0,000 & 0,000 & 0,000 \\
\hline
\end{tabular}

clave de experiencia valiosa, proporciona un punto de partida novedoso desde el que analizar los beneficios del ocio.

Tres son los tipos de beneficios principales que la población joven española obtiene de su ocio significativo: emocionales, sociales y cognitivos. Su ocio más significativo es, para este colectivo, fuente inagotable de beneficios emocionales. Estos beneficios, asociados a la satisfacción de realizar aquello que realmente les gusta, revelan la orientación intrínseca de las y los jóvenes españoles hacia su ocio, cuya práctica constituye en sí misma un beneficio. Los beneficios emocionales son los que presentan puntuaciones más altas y su protagonismo no varía en función de las variables analizadas. El ocio juvenil más sig- 
nificativo proporciona además una alta dosis de beneficios sociales y cognitivos. Ambos tipos de beneficios han sido destacados en la literatura por ser elementos indispensables para el ajuste psicológico y la formación de la identidad que conlleva el desarrollo juvenil (Zarret, Fay, Carrano, Phelps y Letner, 2009).

Las tres prácticas de ocio joven más significativas son, según su importancia, el ocio físico-deportivo, las actividades culturales y las fiestas y celebraciones. Las actividades físico-deportivas son las que reportan una mayor percepción de beneficios, principalmente de tipo físico, emocional, así como la adquisición de destrezas y habilidades técnicas. Resultados similares se obtuvieron en otros estudios como el realizado por Ponce de León, Sanz y Valdemoros (2015). Así, el ocio físico-deportivo no es solo la práctica más significativa, sino también la que les proporciona un abanico más amplio de retornos positivos. Las fiestas y celebraciones, tercer tipo de actividad preferida, les proporcionan beneficios emocionales y sociales, esenciales en esta etapa vital. Sin embargo, llama la atención que las actividades culturales, elegidas por el colectivo joven como uno de sus ocios prioritarios, sea una fuente de beneficios más comedida que otras actividades menos significativas. El ocio cultural reporta, sobre todo, beneficios emocionales y cognitivos, pero en menor medida que prácticas como el turismo/excursionismo o el voluntariado/asociacionismo, actividades que resultan prioritarias para un porcentaje menor de jóvenes y que, sin embargo, para el escaso porcentaje que las practica son fuente de importantes beneficios emocionales, cognitivos y especialmente, sociales. En cuanto al ocio digital, cabe reseñar que, a pesar de ser una práctica muy extendida entre la población joven (Colás, González y de Pablos, 2013), este estudio evidencia que no ocupa un lugar privilegiado entre las prácticas de ocio más significativas de este colectivo (Viñals y Cuenca, 2016) y que la puntuación media de los beneficios que reporta tampoco se halla entre las más altas $(3,09)$. Tales resultados evidencian una aparente contradicción en la que se hace necesario profundizar para esclarecer los motivos de la elevada penetración social de este tipo de ocio. ¿Por qué una actividad de ocio que no es especialmente significativa para las y los jóvenes constituye una práctica cotidiana, plenamente instaurada en su rutina diaria? Aún más, ¿qué factores explican la expansión y persistencia de este hábito si los beneficios que reporta no son los más destacables?

El género emerge como una variable moduladora de los beneficios de ocio juvenil. Los resultados confirman, en primer lugar, que las mujeres muestran, en todos los tipos de beneficio, menores niveles de percepción que los chicos; en segundo lugar, que los tipos de beneficios percibidos por chicos y chicas son ligeramente distintos. Las chicas afirman obtener de su ocio, en menor medida 
que los chicos, destrezas y habilidades técnicas. Lo mismo sucede con los beneficios físicos, ambos tipos de beneficios, fuertemente asociados al ocio físico-deportivo. Sin embargo, las puntuaciones medias se equiparan cuando se trata de beneficios emocionales, lo que significa que tanto chicos como chicas disfrutan de un ocio altamente satisfactorio. Estos resultados apuntan, como lo hacen estudios previos (Barnett, 2013; Codina, Pestana, Castillo y Balaguer, 2016), la existencia de diferencias importantes en torno al ocio de chicos y chicas (lo que les resulta más significativo, el tiempo que le dedican, la frecuencia o compañía con la que lo hacen, etc.). No obstante, siguiendo la estela de pensamiento de Henderson (2009), se confirma que para avanzar en la comprensión del papel del género en el ocio juvenil resulta necesario superar el tratamiento habitual de este aspecto (género) como variable independiente y profundizar, desde enfoques más neutros, en los factores implicados en la construcción social del género y, que probablemente, puedan explicar estas diferencias.

Los resultados obtenidos avalan la idea de que ciertos espacios resultan especialmente propicios para el logro de determinado tipo de beneficios, en consonancia con las actividades que acogen. Así los espacios públicos abiertos congregan, especialmente, el ocio físico-deportivo y las fiestas populares. Las plazas, parques y calles son también espacios idóneos para estar con las amistades, una de las actividades de ocio que mejor representa la categoría "Otras actividades". Todo ello explica por qué este tipo de espacios está asociado a beneficios emocionales y sociales. Los espacios cerrados ajenos, tales como polideportivos, gimnasios, cines, ludotecas, centros culturales, discotecas y bares, acogen casi la mitad de las actividades de ocio físico-deportivo juveniles, así como buena parte de sus actividades culturales. De ahí que, además de altos niveles de beneficios emocionales y sociales, estos espacios se muestren asociados a la percepción de beneficios físicos y adquisición de destrezas y habilidades técnicas. Finalmente, los espacios cerrados propios (la casa, locales privados o lonjas), que acogen buena parte de las actividades culturales juveniles y, en menor medida, fiestas o celebraciones de índole privada parecen ser fuente principal de beneficios emocionales y sociales, fundamentalmente. La literatura consultada subraya que la población joven prefiere y se siente más satisfecha en espacios de ocio que le garanticen cierta autonomía del mundo adulto. Los resultados de este estudio coinciden con los ya existentes (AbbotChampan y Robertson, 2001) y apuntan que la satisfacción (beneficios emocionales) de este colectivo es alta en todos los tipos de espacios, no solo en los cerrados particulares (a priori, especialmente proclives a la autogestión), pero además, permiten constatar que estar satisfecho/a con los espacios cerrados 
particulares no implica necesariamente que en ellos se obtengan altos niveles de otros tipos de beneficios. De hecho, este estudio confirma que la mayor percepción de beneficios se da en los espacios cerrados ajenos, en los que se realizan actividades de ocio preferentemente organizado y que es, precisamente, el tipo de ocio que emerge como principal fuente de beneficio.

Efectivamente, el carácter organizado o no organizado del ocio juvenil se revela como una variable para la consecución de beneficios a través del ocio. Más de la mitad (6 de cada 10) de las actividades de ocio significativas para las y los jóvenes españoles son realizadas al margen de cualquier entidad. La importancia de este dato se evidencia al confirmar que la percepción de beneficios es mayor cuando se trata de formas de ocio organizado. Esta diferencia en términos de beneficios a favor del ocio organizado explica por qué las actividades físico-deportivas y el asociacionismo, que son las que más frecuentemente se realizan en un marco organizado, son también las que proporcionan niveles más altos de beneficios, mientras que el ocio digital y las actividades culturales, cuya práctica se realiza en mayor medida, de forma no organizada, son las que muestran medias de beneficios más bajas en todos los tipos de beneficios, excepto en los emocionales. Estos resultados coinciden con los obtenidos en estudios precedentes (Codina, Pestana, Castillo y Balaguer, 2016; Parra, Oliva y Antolín, 2009; Ramos, Rivera y Moreno, 2012) en los que se confirma que el ocio organizado incentiva el desarrollo juvenil por los numerosos beneficios que desencadena (experiencias enriquecedoras, mayor ajuste emocional, desarrollo de la iniciativa personal y cultivo de valores como la responsabilidad, la superación o el respeto a la diversidad) (Mahoney, Larson, Eccles y Lord, 2005; Parra, Oliva y Antolín, 2009; Sibthorp, Bialeschki, Morgan y Browne, 2013). La literatura científica ratifica, por tanto, el interés de que la población joven se implique en prácticas de ocio organizado pues con ello se propicia un mayor logro de beneficios de diversa índole. Sin embargo, estos datos han de interpretarse con cautela, tomando conciencia de que las investigaciones realizadas al respecto se limitan al ocio organizado. No se tiene constancia de estudios que hayan revisado los retornos positivos del ocio juvenil no organizado. En este artículo son las y los propios jóvenes quienes anuncian que su ocio organizado es más fructífero, en términos de beneficios, que el que realizan por su cuenta. Ahora bien, lejos de descartar las bondades del ocio no organizado ante la evidencia de los datos, nos reafirmamos en la necesidad de continuar estudiando el ocio no organizado para esclarecer la naturaleza o condiciones en las que se optimiza la contribución del ocio no organizado al desarrollo juvenil. 


\section{BIBLIOGRAFÍA}

Abbott-Chapman, J. y Robertson, M. (2001). Youth, Leisure and Home: Space, Place and Identity. Loisir et Société /Society and Leisure, 24(2), 485-506.

Ahedo, R. y Macua, A. (2016). Características de las prácticas de ocio físico-deportivas significativas de los jóvenes españoles. Revista de Psicología del Deporte, 25(2), 67-72.

Barnett, L.A. (2013). What people want from their leisure. Journal of Leisure Research, 45 (2), 150-191.

Broh, B. A. (2002). Linking extracurricular programming to academic achievement: Who benefits and why? Sociology of Education, 75, 69-96.

Chawla, L. (2015). Benefits of nature contact for children. Journal of Planning Literature, 30, 433-452.

Codina, N., Pestana, J.V., Castillo, I. y Balaguer, I. (2016). "Ellas a estudiar y bailar, ellos a hacer deporte: Un estudio de las actividades extraescolares de los adolescentes mediante los presupuestos de tiempo. Cuadernos de Psicología del Deporte, 6(1), 233-242.

Colás, P., González, T. y De Pablos, J. (2013). Juventud y redes sociales: motivaciones y usos preferentes. Comunicar, 40 (XX), 15-23.

Cuenca, M. (2015). Ocio valioso. Documentos de Estudios de Ocio, núm. 52. Bilbao: Universidad de Deusto.

Damon, W. (2004). What is positive youth development? Annals of the American Academy of Political and Social Science, 591, 13-24.

Devine, M.A. y Parr, M. (2008). 'Come on in, but not too Far:' Social Capital in an Inclusive Leisure Setting. Leisure Sciences, 30(5), 391-408.

Doistúa, J. y Ried, A. (2016). Ocio en la naturaleza como espacio de desarrollo juvenil. Revista de Psicología del Deporte, 25(3), 39-44.

Driver, B. L. y Bruns, D. H. (1999). Concepts and uses of the benefits approach to leisure. En E. L. Jackson y T.L. Burton, Leisure studies: prospects for the 21st century. State College, PA: Venture Publishing, 349-369.

Driver, B.L., Brown, P.J. y Peterson, G.L. (1991). Benefits of Leisure. State College, PA: Venture Publishing.

Eccles, J. y Gootman, J. (Eds). (2002). Community programs to promote youth development. Washington, D.C.: National Academy Press.

Fraguela, R., Varela, L. y Sanz, E. (2016). Ocio deportivo, imagen corporal y satisfacción vital en jóvenes españoles. Revista de Psicología del Deporte, 25(2), 33-38.

Gardner, M., Roth, J. y Brooks-Gunn, J. (2008). Adolescents' participation in organized activities and developmental success two and eight years after high school: Do sponsorship, duration, and intensity matter?. Developmental Psychology, 44, 814-830.

Gibson, H. y Chang, S. (2013). Cycling in Mid and Later Life: Involvement and Benefits Sought from a Bicycle Tour. Journal of Leisure Research, 44 (1), 23-51.

Henderson, K. A. (2009). Just research and physical activity: Diversity is more than an independent variable. Leisure Sciences, 31(1), 100-105. 
Kleiber, D.A., Bayón, F, Cuenca, J. y Monteagudo, M. J. (2014). La contribución del ocio experiencial valioso al envejecimiento satisfactorio: estudio de los estilos de ocio de las personas mayores de Euskadi. Inguruak: Revista vasca de sociología y ciencia política, 57-58, 2347-2364.

Kleiber, D. A., Walker, G. y Mannell, R. C. (2011). A social psychology of leisure (2nd ed.). State College, PA: Venture Publishing.

Larson R., Jarrett, R., Hansen, D., Pearce, N., Sullivan, P., Walker, K., Watkins, N. y Wood, D. (2004). Organized youth activities as contexts of positive development. En P. A. Linley y S. Joseph (eds.) Positive psychology in practice. New York: John Wiley \& Sons, 540-560.

Larson, R. W. (2000). Toward a psychology of positive youth development. The American Psychologist, 55, 170-183.

Mahoney, J. L., Larson, R. W. y Eccles, J. S. (2005). Organized activities as contexts of development: Extracurricular activities, after-school and community programs. Hillsdale, NJ: Lawrence Erlbaum Associates.

Mahoney, J. L., Larson, R. W., Eccles, J. S. y Lord, D. (2005). Organized activities as developmental context for children and adolescents. En J. L. Mahoney, R. W. Larson y J. S. Eccles (Eds.). Organized activities as a contexts of development: Extracurricular activities, after-school and community programs. Hillsdale, NJ: Lawrence Erlbaum Associates, 3-22.

Monteagudo, M.J. (2004). Los beneficios del ocio: qué son y para qué sirven. ADOZ. Revista de Estudios de Ocio, 28,5563-72.

Monteagudo, M. J. (2008). Reconstruyendo la experiencia de ocio: características, condiciones de posibilidad y amenazas en la sociedad de consumo. En M. J. Monteagudo (ed.) La experiencia de ocio: una mirada científica desde los Estudios de Ocio. Bilbao: Universidad de Deusto, 81-110.

Monteagudo, M. J., Ahedo, R., Richter, F. y Doistua, J. (2014). Repensando el deporte escolar desde nuevos parámetros. En C. Ortega y F. Bayón (coords.) El papel del ocio en la construcción social del joven. Bilbao: Universidad de Deusto, 197-209.

Nussbaum, M. (2012). Crear capacidades: propuesta para el desarrollo humano. Barcelona: Paidós.

Oliva, A., Pertegal, M.A., Antolín, L., Reina, MC. y Ríos, M. (2011). El desarrollo positivo adolescente y los activos que lo promueven. Un estudio en centros docentes andaluces. Sevilla: Junta de Andalucía. Consejería de Salud.

Ortega, C., Lazcano, I. y Baptista, M. (2015). Espacios de ocio para jóvenes, de la monitorización a la autogestión. Pedagogía Social, Revista Interuniversitaria, 25, 69-89.

Parra, A., Oliva, A. y Antolín, L. (2009). Los programas extraescolares como recurso para fomentar el desarrollo positivo adolescente. Papeles del Psicólogo, 30 (3), 265-275.

Ponce de León, A., Sanz, E., y Valdemoros, M. Á. (2015). Ocio familiar y actividad física en estudiantes de bachillerato: ¿Alianza, rivalidad o independencia? Pedagogía Social. Revista Interuniversitaria, 25, 51-68.

Porter H., Iwasaki Y. y Shank J. (2010). Conceptualizing meaning-making through leisure experiences. Society and Leisure/Loisir et Societe, 33(2), 167-194. 
Ramos, P., Rivera, F. y Moreno, C. (2012). Beneficios del contexto asociativo en las actividades de tiempo libre de los adolescentes españoles. Infancia y Aprendizaje, 35(3), 365-378.

Roth, J. L. y Brooks-Gunn, J. (2003). What exactly is a youth development program? Answers from research and practice. Applied Developmental Science, 7, 94-111.

Sibthorp, J., Bialeschki, M.D., Morgan, C. y Browne, L. (2013). Validating, norming, and utility of a youth outcomes battery for recreation programs and camps. Journal of Leisure Research, 45(4), 514-536.

Tejerina, B., Carbajo, D. y Martínez, M. (2012). El fenómeno de las lonjas juveniles. Nuevos espacios de ocio y socialidad en Vitoria-Gasteiz. Informes del CEIC, 004.

Valdemoros, M, A., Ponce de León, A. y Gradaille, R. (2016). Actividad Física de Ocio Juvenil y Desarrollo Humano. Revista de Psicología del Deporte, 25(2), 45-51.

Viñals, A. y Cuenca, J. (2016) Ocio entre pares en la era digital: percepción del ocio conectado juvenil. Revista de Psicología del Deporte, 25(2), 61-65.

Zarret, N., Fay, K., Li, Y., Carrano, J., Phelps, E. y Letner, R. M. (2009). More than child's play: variable and pattern-centered approaches for examining effects of sports participation on youth development. Development Psychology, 45, 368-382.

Zeijl, E., Du Bois-Reymond, M. y Te Poel, Y. (2001). Young Adolescents' Leisure Patterns, Society and Leisure, 24(2), 379-402.

MARÍA JESÚS MONTEAGUDO SÁNCHEZ. Doctora en Ocio y Potencial Humano y Licenciada en Psicología por la Universidad de Deusto. Investigadora asociada en el Instituto de Estudios de Ocio y miembro del equipo de Investigación "Ocio y Desarrollo Humano" de la Universidad de Deusto, reconocido por Gobierno Vasco en categoría A (IT984-16). Su investigación se centra en el estudio del ocio como factor de desarrollo humano, el ocio a lo largo de la vida, beneficios y barreras para la participación así como en el ocio deportivo. Es autora de una decena de monografías, más de 25 capítulos de libro y casi una veintena de artículos en revistas especializadas de alto impacto. Ha sido IP de casi una veintena de investigaciones y ha participado como investigadora en 36 proyectos estatales e internacionales.

orcid.org/0000-0002-6725-5265. Email: mjmonte@deusto.es Correspondencia: María Jesús Monteagudo. Avda. universidades 24, 48007 Bilbao. Bizkaia. España.

RUTH AHEDO GONZÁLEZ: Licenciada en Psicología por la Universidad de Deusto y Doctoranda en el programa Ocio, Cultura y Comunicación para el Desarrollo Humano. Es miembro del Instituto de Estudios de Ocio y forma parte del equipo de Investigación Ocio y Desarrollo Humano de la Universidad de 
Deusto. Vinculada a este equipo ha desarrollado numerosas investigaciones, entre ellas, los Planes Nacionales RESORTES (2013-2015) e ITINERE (20132015), así como el proyecto europeo CONNECT. Connecting Audiences (20172019). orcid.org/0000-0002-4416-5187. Email: ruth.ahedo@deusto.es

ANA PONCE DE LEÓN ELIZONDO: Catedrática de la Universidad de La Rioja. Doctora en Ciencias de la Educación, Premio Extraordinario Doctorado (1996). Directora del Grupo de Investigación Actividad Física y Deporte en el espacio y tiempo de Ocio [AFYDO] de la UR y miembro fundador de la Red de Investigación OcioGune. Ha sido Investigadora Principal en 19 proyectos de I+D+I en temas de Educación, Ocio, Actividad Físico-deportiva y Familia; ha dirigido 11 tesis doctorales y es autora de más de un centenar de libros, capítulos de libro y artículos científicos de alto prestigio e impacto internacional integrados en sistemas de indización internacionales como Social Science Citation Index, JCR, Scopus, Erih, Inrecs, Isoc, Latindex, Dice, Recyt, Redalic. orcid.org/0000-0003-4622-8062. Email: ana.ponce@unirioja.es

Recibido: 03/04/2017

Aceptado: 16/09/2017 